\title{
A Path to Precision Medicine: Incorporating Blood-Based Biomarkers in Stroke Rehabilitation
}

\author{
Byung-Mo Oh, MD, $\mathrm{PhD}^{1,2}$
}

\begin{abstract}
${ }^{1}$ Department of Rehabilitation Medicine, Seoul National University Hospital, Seoul National University College of Medicine, Seoul;
${ }^{2}$ National Traffic Injury Rehabilitation Hospital, Yangpyeong, Korea
\end{abstract}

Biomarkers are biological variables that can be measured and quantified and which provide valuable information about the physiologic state or diseases. Broadly, biomarkers include not only the laboratory results for various specimens but also the findings of functional assessments and imaging tests. The term is also used, in a narrower meaning, to indicate a sign that can be measured from a blood sample. Biomarkers are useful for early diagnosis, differentiation of etiologies, and predicting prognosis in various diseases.

Precision medicine has been proposed as a feature of future medicine. Precision medicine refers to a medical model that provides the most appropriate treatment based on an individual patient's genetics, medical condition, environment, and lifestyle. With the wide range of data for various types of biomarkers, machine learning algorithms capable of processing these data, and the rapid development of computation power, precision medicine has become a popular trend in medicine. However, precision medicine remains in its early stages in the field of rehabilitation medicine. The additional identification and combination of biomarkers, combined with data on disease, patient characteristics, and functional assessment will accelerate the development of precision medicine in rehabilitation therapy.
In the study by Yoon et al. [1] published in this volume, the authors analyzed data from the Korean Stroke Cohort (KOSCO) study and the correlation between the initial blood test results after stroke and the trajectories of functional recovery [2]. They reported that initial hyperglycemia was negatively associated with the ultimate functional recovery even in non-diabetic patients with ischemic stroke.

This finding is consistent with those from previous studies. In 2012, Hasan et al. [3] conducted a systematic review and meta-analysis of 141 clinical studies on 136 biomarkers. They reported that markers showing significant correlations with stroke diagnosis, namely C-reactive protein (CRP), P-selectin, homocysteine, blood glucose, and fibrinogen levels, were also significant markers for the prediction of prognosis using the modified Rankin Scale. Blood glucose level was associated with the risk for clinical worsening within the next 48 hours, the risk for in-hospital mortality, and the risk of hemorrhagic transformation after thrombolytic therapy.

The study by Yoon et al. [1] published in this volume has clinical implications of importance in that it has a higher sample size compared to previous studies and included a relatively milder group of participants who did not die during the study period. Thus, the results of this

Received October 18, 2021; Accepted October 25, 2021; Published online October 31, 2021

Corresponding author: Byung-Mo Oh

Department of Rehabilitation Medicine, Seoul National University Hospital, Seoul National University College of Medicine, 101 Daehak-ro, Jongno-gu, Seoul 03080, Korea. Tel: +82-2-2072-2619, Fax:+82-2-6072-5244, E-mail: moya1@snu.ac.kr ORCID: Byung-Mo Oh (https://orcid.org/0000-0001-9353-7541).

(c) This is an open-access article distributed under the terms of the Creative Commons Attribution Non-Commercial License (http://creativecommons.org/ licenses/by-nc/4.0) which permits unrestricted noncommercial use, distribution, and reproduction in any medium, provided the original work is properly cited. Copyright (c) 2021 by Korean Academy of Rehabilitation Medicine 


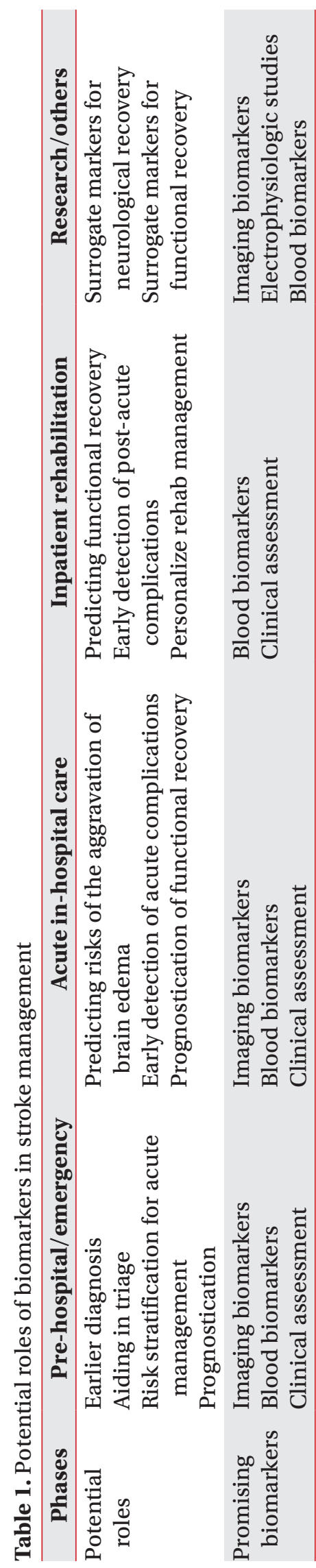

study demonstrated the potential utility of blood-based biomarkers in the prediction of long-term functional outcome after stroke.

\section{POTENTIAL USE SCENARIOS OF BLOOD-BASED BIOMARKERS IN STROKE CARE}

Biomarkers have various potential uses in stroke management [4] (Table 1).

The actively investigated areas for the application of biomarkers include the use of blood biomarkers for the early diagnosis of stroke and determining the status of its emergency. For example, blood levels of creatine kinase MB (CK-MB) fraction and troponin have been evaluated for the early diagnosis of myocardial infarction. However, compared to myocardial infarction, no effective biomarkers have been validated for stroke. For early diagnosis, biomarker levels must increase in the blood early after stroke and be specific to not only brain tissues but also the pathophysiology of stroke. However, it is difficult to identify the increase in blood concentrations of brain-specific biomarkers at early phases due to the blood-brain barrier, which continues to be a limitation. Furthermore, clinically, as diffusion-weighted magnetic resonance imaging has high imaging speed; and high sensitivity and specificity, it is difficult for novel biomarkers to replace this diagnostic modality.

However, biomarkers provide additional information that can assist with more rapid and accurate decisionmaking for disease management. For example, information from biomarker panels obtained at admission accelerated decision-making for the use of thrombolytics [5]. Biomarkers can also be useful in rehabilitation to predict the response to specific treatments or for stratification based on the risk of complications.

Studies have investigated the potential of blood biomarkers for the prediction of functional prognosis after stroke. For example, fibrinogen [6] and CRP [7] levels are associated with a poor prognosis. A systematic review by Whiteley et al. [8] in 2009 indicated that while some biomarkers were consistently correlated with stroke prognosis in studies published between 1996 and 2007, these did not increase the predictability in clinical settings.

In their 2012 prospective study using various blood biomarkers to predict the prognosis of 270 stroke patients, Whiteley et al. [9] measured blood biomarker concentra- 
tions within 24 hours of the onset of ischemic stroke and evaluated mortality rate or correlation with disability after 3 months. While they reported a significant correlation between interleukin- 6 and $\mathrm{N}$-terminal-pro hormone B-type natriuretic peptide levels, the predictability did not increase for these two biomarkers compared to prognosis prediction based on the clinical information. Thus, while biomarkers significantly associated with poor prognosis have been identified, superior clinical prediction models are already available, namely the National Institutes of Health Stroke Scale and age.

\section{LIMITATIONS AND THE FUTURE OF BIOMARKERS IN STROKE REHABILITATION}

In consideration of the complex pathophysiology of stroke and resulting disabilities, there are clear limitations of blood biomarkers. First, biomarkers do not provide information on the lesion location. Even if the infarct volume may be the same, the functional prognosis differs greatly between those occurring in the parietal association area or the internal capsule posterior limb. However, it is difficult to predict this differential information based only on blood biomarker concentrations. Second, the blood-brain barrier results in a significant delay in the expression of brain tissue-specific biomarkers in the blood. Third, study results indicate that it is difficult for a single biomarker to have sufficient sensitivity and specificity for clinical applications. Therefore, approaches for biomarker panels using multiple important biomarkers will be required.

Unfortunately, there have been very little literature on biomarkers related to stroke rehabilitation. Clinical and neurophysiological measurements, as well as imaging study results, have previously been used to predict patients' functional recovery $[10,11]$. In order to prepare for the coming era of precision medicine, it is essential to not only adequately evaluate and quantify various functional outcomes in detail but also to understand the biological changes that occur at the beginning of the disease and throughout rehabilitation therapy. For this reason, prospective clinical studies that not only evaluate the functional level of patients but also collect blood biomarkers should be actively conducted. It would be ideal to form a multicenter research consortium including investigators from various areas. Furthermore, large-scale studies such as the KOSCO project need to fully consider, from the planning stage, whether to include blood biomarker collection.

\section{CONFLICT OF INTEREST}

No potential conflict of interest relevant to this article was reported.

\section{REFERENCES}

1. Yoon JA, Shin YI, Kim DY, Sohn MK, Lee J, Lee SG, et al. Post-stroke hyperglycemia in non-diabetic ischemic stroke is related with worse functional outcome: a cohort study. Ann Rehabil Med 2021;45:359-367.

2. Kim BR, Lee J, Sohn MK, Kim DY, Lee SG, Shin YI, et al. Risk factors and functional impact of medical complications in stroke. Ann Rehabil Med 2017;41:753-60.

3. Hasan N, McColgan P, Bentley P, Edwards RJ, Sharma P. Towards the identification of blood biomarkers for acute stroke in humans: a comprehensive systematic review. Br J Clin Pharmacol 2012;74:230-40.

4. Kernagis DN, Laskowitz DT. Evolving role of biomarkers in acute cerebrovascular disease. Ann Neurol 2012;71:289-303.

5. Ribo M, Montaner J, Molina CA, Arenillas JF, Santamarina E, Quintana M, et al. Admission fibrinolytic profile is associated with symptomatic hemorrhagic transformation in stroke patients treated with tissue plasminogen activator. Stroke 2004;35:2123-7.

6. del Zoppo GJ, Levy DE, Wasiewski WW, Pancioli AM, Demchuk AM, Trammel J, et al. Hyperfibrinogenemia and functional outcome from acute ischemic stroke. Stroke 2009;40:1687-91.

7. Welsh P, Barber M, Langhorne P, Rumley A, Lowe GD, Stott DJ. Associations of inflammatory and haemostatic biomarkers with poor outcome in acute ischaemic stroke. Cerebrovasc Dis 2009;27:247-53.

8. Whiteley W, Chong WL, Sengupta A, Sandercock P. Blood markers for the prognosis of ischemic stroke: a systematic review. Stroke 2009;40:e380-9.

9. Whiteley W, Wardlaw J, Dennis M, Lowe G, Rumley A, Sattar N, et al. The use of blood biomarkers to predict poor outcome after acute transient ischemic attack or ischemic stroke. Stroke 2012;43:86-91.

10. Park JG, Lee KW, Kim SB, Lee JH, Kim YH. Effect of de- 
creased skeletal muscle index and hand grip strength on functional recovery in subacute ambulatory stroke patients. Ann Rehabil Med 2019;43:535-43.

11. Smith MC, Ackerley SJ, Barber PA, Byblow WD, Stin- ear CM. PREP2 algorithm predictions are correct at 2 years poststroke for most patients. Neurorehabil Neural Repair 2019;33:635-42. 